\title{
Un caso di insufficienza renale acuta e miopatia reversibili
}

\author{
A. Federico' ${ }^{1}$, D. Ricciardi ${ }^{1}$, D. Rossi ${ }^{2}$, F. Bruni ${ }^{1}$ \\ 1U.O. N efrologia e Dialisi, ASUR M arche Zona Territoriale n. 12, San Benedetto del Tronto (Ascoli Piceno) \\ 2U.O. Nefrologia e Dialisi, USL 2, Assisi (Perugia)
}

\section{Presentazione del Caso Clinico}

U na donna di razza caucasica di 51 anni, fumatrice, eseguiva una visita nefrologica presso il nostro ambulatorio per il recente riscontro di moderata insufficienza renale (creatinina $1.7 \mathrm{mg} / \mathrm{dL}$ ). La paziente lamentava da circa 4 mesi una sintomatologia caratterizzata da incremento ponderale con edemi prevalentemente localizzati alle labbra e alle estremità, astenia, malessere generale; segnalava inoltre la comparsa di amenor rea da alcuni mesi. In anamnesi patologica remota riferiva solamente sindrome eclamptica in occasione della prima gravidanza. Negava disturbi urinari, lamentava stipsi ostinata e crampi muscolari. Precedenti esami ematobiochimici mostravano una funzione renale normale.

Obiettivamente si riscontrava pallore mucocutaneo, succulenza agli arti inferiori in zona pre-tibiale e in zona periorbitaria, bradicardia sinusale. $\mathrm{N}$ on evidenza di gozzo tiroideo, né di linfoadenopatie superficiali. Non altri reperti degni di nota a carico degli altri organi e apparati. Peso corporeo $68 \mathrm{~kg}$, altezza $1.70 \mathrm{~cm}$ valori pressori pari a $125 / 80 \mathrm{mmHg}$. La diuresi era conser vata.

Gli esami di laboratorio mostravano lieve anemia normocromica normocitica ( $\mathrm{Hb} 11.9 \mathrm{~g} / \mathrm{dL}, \mathrm{MCV} 94 \mathrm{fl}, \mathrm{MCH}$ $31 \mathrm{pg}$ ), moderata insufficienza renale (creatinina 1.7 $\mathrm{mg} / \mathrm{dL}$, clearance della creatinina endogena calcolata con la formula di Cockroft-Gault pari a $42 \mathrm{ml} / \mathrm{min} / 1,73$ $\mathrm{mq}$ ), ipercolesterolemia ( $308 \mathrm{mg} / \mathrm{dL}$ ).

In ambulatorio la paziente eseguiva un ulteriore controllo laboratoristico, che, oltre a confermare i dati precedenti, evidenziava: incremento dei valori di GOT ( 94 $\mathrm{U} / \mathrm{I})$ con normalità dei valori di GPT (28 U/I), aumento dei livelli di CPK totale ( $3600 \mathrm{U} / \mathrm{I}$ ), di mioglobina ( 335.7 $\mathrm{ng} / \mathrm{mL}$ ) e di LDH (1043 U/I), kaliemia ai limiti alti della norma ( $5.2 \mathrm{mE} \mathrm{q/} \mathrm{L).} \mathrm{L'esame} \mathrm{urine} \mathrm{standard} \mathrm{evidenziava}$ positività al dipstick per sangue e per proteine in tracce, al sedimento urinario non erano presenti globuli rossi. Tali reperti sono stati considerati compatibili con la presenza di mioglobinuria. Nella norma lo screening immunologico (VES, PCR, ANA, ENA).

Un esame ECG evidenziava bradicardia sinusale (FC 55 bpm), basso voltaggio dei complessi QRS, onda T ischemica nelle derivazioni anterolaterali. Un'ecografia renale evidenziava reni normali per sede, forma e dimensioni; il parenchima era ben rappresentato con corticale di normale spessore ed ecogenicità; buona la differenziazione corticomidollare; non immagini ecografiche riferibili a calcoli, non dilatazione delle vie escretrici superiori.

\section{Diagnosi differenziale}

\section{Cause di rabdomiolisi}

La rabdomiolisi è una sindrome caratterizzata da necrosi muscolare e dal conseguente rilascio di costituenti intramuscolari in circolo. È una condizione che può avere diversi gradi di severità, che vanno dall'incremento asintomatico del valore degli enzimi muscolari a casi gravi, potenzialmente letali, associati ad estremi incrementi del CPK (1). È generalmente associata a mioglobinuria e, se sufficientemente severa, può causare significative alterazioni elettrolitiche e insufficienza renale acuta, anche grave. La sue principali manifestazioni cliniche sono la debolezza muscolare, la mialgia e l'emissione di urine colorate (1).

Le cause più comuni di rabdomiolisi sono l'abuso di alcol (2), I'esercizio muscolare estremo (3), una compressione muscolare protratta (4) el'utilizzo di alcuni farmaci 
o sostanze illecite (5-7); va inoltre tenuta presente la sindrome da schiacciamento (Tab. I).

Generalmente l'eziologia è facilmente desumibile dall'anamnesi e dai segni clinici (trauma, esercizio fisico estremo, inter vento chirurgico ecc.); più raramente il fattore scatenante non è così evidente e vanno ricercate altre cause, quali alterazioni elettrolitiche, infezioni, farmaci, tossine o endocrinopatie. Devono, infine, essere presi in considerazione i difetti genetici enzimatici muscolari in caso di pazienti che presentano episodi di rabdomiolisi ricorrente a partire dall'età infantile, favoriti da fattori scatenanti quali un lieve o moderato esercizio fisico oppure un'infezione virale $(6,8)$.

La nostra paziente presentava un'anamnesi patologica remota negativa per pregressi disturbi muscolari e un'anamnesi familiare negativa per miopatie. Non lamentava mialgia, negava traumi e sforzi muscolari ec- cessivi, come pure l'abuso di alcol, farmaci o droghe. Gli indici di flogosi e lo screening immunologico risultavano nella norma.

In considerazione dei dati anamnestici, clinici e laboratoristici a disposizione, veniva avanzata l'ipotesi diagnostica di una miopatia associata a ipotiroidismo. Pertanto, è stata eseguita la determinazione degli ormoni tiroidei e del TSH che sono risultati come segue: TSH ultrasensibile $>100 \mathrm{mUI} / \mathrm{mL}$ (vn 0.49-4.67), FT3 $1.18 \mathrm{pg} / \mathrm{mL}$ (vn 1.64-3.45), FT4 $<0.40 \mathrm{ng} / \mathrm{dL}$ (vn 0.70-1.85), suggestivi per un quadro di ipotiroidismo primitivo. A completamento la paziente eseguiva il dosaggio degli anticorpi anti-tireoglobulina e anti-perossidasi risultati elevati ( $r i-$ spettivamente $419 \mathrm{UI} / \mathrm{mL}$ (vn 0-115), $4700 \mathrm{UI} / \mathrm{mL}$ (vn $<10)$. Un'ecografia tiroidea evidenziava ghiandola tiroidea in sede e di dimensioni nei limiti, con struttura ghiandolare diffusamente disomogenea; in particolare a

TABE LLA I - CAUSE DI RABDOM IOLISI

\begin{tabular}{|c|c|c|c|c|}
\hline \multirow[t]{4}{*}{ Cause di rabdomiolisi } & $\begin{array}{l}\text { Traumatiche o } \\
\text { da compressione }\end{array}$ & \multicolumn{3}{|c|}{$\begin{array}{l}\text { 1. Trauma multiplo } \\
\text { 2. Chirurgia ortopedica o vascolare } \\
\text { 3. Coma } \\
\text { 4. Immobilizzazione }\end{array}$} \\
\hline & \multirow{3}{*}{$\begin{array}{l}\text { Non } \\
\text { traumatiche }\end{array}$} & & $\begin{array}{l}\text { Non patologia } \\
\text { muscolare }\end{array}$ & $\begin{array}{l}\text { 1. Esercizio fisico estremo } \\
\text { 2. Colpo di calore } \\
\text { 3. Anemia a cellule falciformi } \\
\text { 4. Convulsioni } \\
\text { 5. Stati ipercinetici }\end{array}$ \\
\hline & & $\begin{array}{l}\text { Da sforzo } \\
\text { muscolare }\end{array}$ & $\begin{array}{l}\text { Patologia } \\
\text { muscolare }\end{array}$ & $\begin{array}{l}\text { 1. M iopatie metaboliche: } \\
\text { a. Disordini della glicogenolisi, es M alattia } \\
\text { di M cArdle } \\
\text { b. Disordini della glicolisi } \\
\text { c. Disordini del metabolismo metabolico } \\
\text { d. Disordini del metabolismo delle purine, es. } \\
\text { Distrofia muscolare di Duchenne } \\
\text { e. altre } \\
\text { 2. M iopatie mitocondriali } \\
\text { 3. Ipertermia maligna } \\
\text { 4. Sindrome neurolettica maligna }\end{array}$ \\
\hline & & $\begin{array}{l}\text { In assenza di } \\
\text { sforzo } \\
\text { muscolare }\end{array}$ & & $\begin{array}{l}\text { 1. Alcolismo } \\
\text { 2. Farmaci e tossine: statine, ciclosporina, } \\
\text { itraconazolo, eritromicina, colchicina, } \\
\text { cocaina, anfetamine... } \\
\text { 3. Infezioni: } \\
\text { a. Batteriche: Streptococco, Salmonella, Legionella, } \\
\text { Stafilococco, Listeria } \\
\text { b. Virali: Influenza B, Parainfluenza, adenovirus, } \\
\text { coxsackievirus, echovir us, herpes simplex, } \\
\text { CM V, HIV, E pstein-Barr } \\
\text { 4. Alterazioni elettrolitiche: iponatremia, ipernatremia, } \\
\text { ipokaliemia, ipofosfatemia, ipocalcemia } \\
\text { 5. Endocrinopatie: ipotiroidismo, tireotossicosi, } \\
\text { chetoacidosi diabetica, sindrome iperosmolare non } \\
\text { chetoacidotica } \\
\text { 6. M iopatie infiammatorie: polimiosite, dermatomiosite }\end{array}$ \\
\hline
\end{tabular}


carico di entrambi i lobi tiroidei si osservavano alcune aree ipoecogene a margini sfumati, delle dimensioni massime di $15 \mathrm{~mm}$ circa. I reperti descritti deponevano in prima ipotesi per una tiroidite cronica di tipo autoimmune.

\section{Miopatia in corso di ipotiroidismo}

Disturbi muscolari in corso di ipotiroidismo sono tutt'al tro che infrequenti, anche se il paziente difficilmente riferisce disturbi, quali crampi muscolari, mialgie e debolezza muscolare, sin dall'esordio (9). Lo spettro clinico della miopatia da ipotiroidismo nell'adulto è ampio, anche nell'ambito dello stesso paziente, in momenti diversi del corso della malattia. La durata e la severità dell'ipotiroidismo possono correlare con la presentazione clinica della miopatia.

$\checkmark$ Un aumento dei livelli sierici di CPK si verifica fino al $90 \%$ dei pazienti ipotiroidei. II livello enzimatico non è strettamente correlato con la severità della miopatia, ma può essere condizionato dalla gravità dell'ipotiroidismo (10-13). L'incremento del CPK può precedere anche di alcuni anni le manifestazioni cliniche dell'ipotiroidismo, pertanto, è una condizione che andrebbe esclusa in tutti quei pazienti con incremento di CPK non altrimenti spiegabile, specialmente se anziani, nei quali i sintomi dell'ipotiroidismo possono essere mascherati.

$\checkmark$ All'aumento degli enzimi muscolari, può associarsi mialgia, crampi e debolezza muscolare, spesso esacerbati dall'esercizio fisico (14).

$\checkmark$ Alcuni pazienti presentano un quadro di miopatia prossimale, caratterizzata da debolezza muscolare prossimale simmetrica a sviluppo lentamente progressivo. Le zone più colpite sono quelle dei cingoli scapolari e delle articolazioni dell'anca. Colpisce circa un terzo dei pazienti (7).

$\checkmark$ Occasionalmente, una sindrome simil-polimiosite, con debolezza muscolare prossimale e marcato aumento degli enzimi muscolari, può essere una delle espressioni cliniche della miopatia da ipotiroidismo. Può avere un decorso fulminante con andamento clinico simile a quello della polimiosite $(10,15)$.

$\checkmark$ Una vera e propria rabdomiolisi relata all'ipotiroidismo è infrequente e pochi casi sono stati riportati in letteratura (16). Nella maggior parte dei casi segnalati è descritta la presenza di fattori scatenanti quali traumi, sforzi fisici, terapia ipolipemizzante (17). La miolisi è principalmente causata da alterazioni strutturali delle fibre muscolari, dalla deposizione di glicosaminoglicani, da una ridotta contrattilità delle unità actina-miosina, da un basso turnover dell'ATP nelle fibre muscolari scheletriche e da un'alterazione reversibile nella glicogenolisi $(11,18)$. La rabdomiolisi può diventare una condizione estremamente grave quando è complicata da insufficienza renale acuta severa.

$\checkmark$ In un terzo di pazienti con ipotiroidismo si verifica il cosiddetto "mioedema", ovvero una contrattura localizzata del muscolo scheletrico indotta dalla percussione diretta sul muscolo stesso. II fenomeno, che è tipico della miopatia da ipotiroidismo, dura 30-60 secondi ed è dovuto a una contrazione prolungata associata a un ritardato rilassamento $(14,19)$. Non è patognomonico di una condizione di ipotiroidismo, potendo verificarsi anche in caso di malnutrizione.

$\checkmark$ Infine, si può osser vare una rara condizione, la cosiddetta sindrome di Hoffman, caratterizzata da iper trofia muscolare, debolezza e rallentamento dei movimenti $(14,20)$.

\section{Terapia della miopatia da ipotiroidismo}

La miopatia da ipotiroidismo clinicamente risponde bene al trattamento ormono-sostitutivo. Si assiste, inoltre, a una rapida riduzione dei livelli di CPK con l'avvio della terapia, rientrando entro i valori di norma nell'arco di alcune settimane, generalmente ancor prima che il valore di TSH si normalizzi $(10,12,14,21,22)$. La correzione dell'ipotiroidismo compor ta anche il recupero della funzione renale e la regressione di un'eventuale rabdomiolisi.

I sintomi clinici, quali la debolezza muscolare, possono impiegare più tempo a scomparire, nonostante la completa correzione dell'ipotiroidismo (9).

$\mathrm{N}$ el caso della nostra paziente, è stata subito avviata la terapia ormono-sostitutivo con L-tiroxina ( $100 \mathrm{mcg} / \mathrm{die}$ per os). Al fine di preservare la funzione renale, si è cercato di aumentare la diuresi somministrando liquidi per via parenterale e stimolando la paziente ad aumentarne l'assunzione per via orale. Nei primi giorni, sono stati inoltre somministrati bicarbonati dapprima per via parenterale e successivamente per via orale, al fine di indurre una diuresi alcalina.

A distanza di solo tre settimane di terapia con L-tiroxina, si è ottenuta una parziale correzione dell'ipotiroidismo, il miglioramento clinico (scomparsa degli edemi, delI'astenia e del malessere generale; ripresa del ciclo mestruale), una notevole riduzione dei valori di CPK e I'adeguamento della funzione renale (Tab. II). L'esame urine è attualmente negativo.

\section{Gestione dei pazienti con rabdomiolisi}

La gestione dei pazienti con rabdomiolisi prevede un duplice inter vento: il trattamento della malattia sottostante 
TABELLA || - INDICI DI LABORATORIO DELLA PAZIENTE

\begin{tabular}{lccccc}
\hline & $\begin{array}{c}\mathrm{FT4} \\
\mathrm{ng} / \mathrm{dl} \\
(\mathrm{vn} 0.70-1.85)\end{array}$ & $\begin{array}{c}\mathrm{FT3} \\
\mathrm{pg} / \mathrm{ml} \\
(\mathrm{vn} \mathrm{1.64-3.45)}\end{array}$ & $\begin{array}{c}\mathrm{TSHu} \\
\mathrm{mUl} / \mathrm{ml} \\
(\mathrm{vn} 0.490-4.670)\end{array}$ & $\begin{array}{c}\text { Creatinina } \\
\mathrm{mg} / \mathrm{dl} \\
(\mathrm{vn} \mathrm{0.5-1)}\end{array}$ & $\begin{array}{c}\text { CPK } \\
\text { UI/I } \\
(\mathrm{vn} 25-175)\end{array}$ \\
\hline $\begin{array}{l}\text { Esordio } \\
\text { (dicembre 2007) }\end{array}$ & $<0.40$ & 1.18 & $>100$ & 1.7 & 3600 \\
Dopo 3 settimane di terapia & 1.13 & 2.46 & 25.810 & 0.9 & 419 \\
\hline
\end{tabular}

e l'attuazione di misure atte a prevenire o gestire il danno renale conseguente alla mioglobinuria. Per quanto riguarda il secondo punto, le principali misure terapeutiche consistono nella somministrazione di fluidi, anche nei pazienti non ipovolemici, al fine di minimizzare il danno tubulare renale causato dalla mioglobina. Sono, inoltre, comunemente impiegate l'infusione di mannitolo o la somministrazione di soluzioni alcaline con l'obiettivo di indurre una diuresi con $\mathrm{pH}$ superiore a 6.5. Alcuni studi avrebbero suggerito che il mannitolo potrebbe minimizzare il danno tubulare riducendo la deposizione intra-tubulare del pigmento, agendo come legante dei radicali liberi, riducendo la viscosità ematica e inducendo vasodilatazione renale. La somministrazione di bicarbonato ridurrebbe il rischio di formazioni di cilindri tubulari e quindi il danno renale. Va, tuttavia, segnalato che non esiste tuttora una chiara evidenza che giustifichi l'utilizzo di questi agenti, mancando studi randomizzati controllati. Cautela deve essere infine utilizzata in caso di somministrazione di discrete quantità di bicarbonato, potendo indurre o peggiorare un'ipocalcemia $(1,6)$.

\section{Conclusioni}

In conclusione, la nostra paziente ha presentato un quadro di insufficienza renale acuta secondaria a miopatia da ipotiroidismo primitivo misconosciuto. La rabdomiolisi è una potenziale complicanza della miopatia da ipotiroidismo, in genere favorita da fattori precipitanti. La nostra paziente non presentava altre apparenti cause o fattori precipitanti di aumento del CPK.

Infine, va altresì tenuto presente che l'ipotiroidismo, indipendentemente dalla presenza o meno di una miopatia, può essere di per sé causa di insufficienza renale di nuova insorgenza, oltre che di un peggioramento di un'insufficienza renale cronica pre-esistente, potendo assistere a un recupero fino al $50 \%$ della funzione renale una volta iniziata la terapia sostitutiva (23-25). In tal caso, I'aumento dei livelli sierici di creatinina riconosce varie cause, tra cui predominano gli effetti emodinamici e un'azione diretta sul rene degli ormoni tiroidei (26).

e-mail: federicoantonio1@gmail.com

\section{B ibliografia}

1. Huerta-Alardín AL, Varon J, M arik PE. Bench-to-bedside review: Rhabdomyolysis - an over view for clinicians. Critical Care 2005; 9 (2): 158-69.

2. Bessa $0 \mathrm{Jr}$. Alcoholic rhabdomyolysis: a review. Conn M ed 1995; 59 (9): 519-21.

3. Line RL, Rust GS. Acute exertional rhabdomyolysis. Am Fam Physician 51995; 2 (2): 502-6.

4. Biswas S, Gnanasekaran I, I vatury RR, Simon R, Patel AN . Exaggerated lithotomy position-related rhabdomyolysis. Am Surg 1997; 63 (4): 361-4.

5. Alejandro DS, Petersen J. M yoglobinuric acute renal failure in a cardiac transplant patient taking lovastatin and cyclosporine. J Am Soc Nephrol 1994; 5 (2): 153-60.

6. Sauret J M, M arinides G, Wang GK. Rhabdomyolysis. Am Fam Physician 2002; 65 (5): 907-12.

7. Horowitz BZ, Panacek EA, Jouriles NJ. Severe rhabdomyolysis with renal failure after intranasal cocaine use. J Emerg M ed 1997; 15 (6): 833-7.

8. Warren JD, Blumbergs PC, Thompson PD. RhabdomyoIysis: a review. M uscle Nerve 2002; 25: 332.

9. Duyff RF, Van den B osch J, Laman DM, van Loon BJ, Linssen WH. N euromuscular findings in thyroid dysfunction: 
a prospective clinical and electrodiagnostic study. J Neurol Neurosurg Psychiatry 2000; 68: 750.

10. M adariaga, M G. Polymyositis-like syndrome in hypothyroidism: review of cases reported over the past twentyfive years. Thyroid 2002; 12: 331.

11. M cKeran RO, Slavin G, Ward P, Paul E, M air WG. Hypothyroid myopathy. A clinical and pathological study. J Pathol 1980; 132 (1): 35-54.

12. Giampietro O, Clerico A, Buzzigoli G, Del Chicca M G, Boni C, Carpi A. Detection of hypothyroid myopathy by measurements of various serum muscle markers- myoglobin, creatine kinase, lactate dehydrogenase and their isoenzymes. Correlations with thyroid hormone levels (free and total) and clinical usefulness. Horm Res 1984; 19 (4): 232-42.

13. Kar PM, Hirani A, Allen MJ. Acute renal failure in a hypothyroid patient with rhabdomyolysis. Clin Nephrol 2003; 60 (6) : 428-9.

14. Kendall-Taylor P, Turnbull DM . Endocrine myopathies. Br M ed J (Clin Res Ed) 1983; 287 (6394): 705-8.

15. Hochberg M C, Koppes GM, Edwards CQ, Barnes HV, Arnett FC J r. Hypothyroidism presenting as a polymyositislike syndrome. Report of two cases. Arthritis Rheum 1976; 19 (6) : 1363-6.

16. Altay $M$, Duranay $M$, Ceri $M$. Rhabdomyolysis due to hypothyroidism. Nephrol Dial Transplant 2005; 20 (4) : 847-8.

17. Kursat S, Alici T, Colak HB. A case of rhabdomyolysis induced acute renal failure secondary to statin-fibratederivative combination and occult hypothyroidism. Clin Nephrol 2005; 64 (5): 391-3.

18. Wiles CM, Young A, J ones DA, E dward RHT. M uscle re- laxation rate, fibre type composition and energy turnover in hyper and hypothyroid patients. Clin Sci 1979; 57: 375-84.

19. M izusawa H, Takagi A, Nonaka I, Sugita H, Toyokura Y. M uscolar abnormalities in experimental hypotyroidism of rats with special reference to the mounding phenomenon. Exp Neurol 1984; 85 (3): 480-92.

20. Klein I, Parker M, Shebert R, Ayyar DR, Levey GS. Hypothyroidism presenting as muscle stiffness and pseudohypertrophy: Hoffmann's syndrome. Am J M ed 1981; 70 (4): 891-4.

21. Klein I, M antell P, Parker M , Levey GS. Resolution of abnormal muscle enzyme studies in hypothyroidism. Am J M ed Sci 1980; 279: 159.

22. Khaleeli AA, Edwards RH. Effect of treatment on skeletal muscle dysfunction in hypothyroidism. Cli Sci (Lond) 1984; 66: 63.

23. Hlad CJ Jr, Bricker NS. Renal function and I 131 clearance in hyperthyroidism and myxedema. J Clin Endocrinol M etab 1954; 14 (12): 1539-50.

24. Al-Fifi S, Girardin C, Sharma A, Rodd C. M oderate renal failure in association with prolonged acquired hypothyroidism in children. Acta Paediatr 1999; 88 (7): 715-7.

25. M akino $Y$, Fujii T, Kuroda S, Inenaga T, Kawano Y, Takishita S. Exacerbation of renal failure due to hypothyroidism in a patient with ischemic nephropathy. Nephron 2000; 84 (3) : 267-9.

26. van Welsem M E, Lobatto S. Treatment of severe hypothyroidism in a patient with progressive renal failure leads to significant improvement of renal function. Clin Nephrol 2007; 67 (6) : 391-3. 